\title{
miR-92a regulates the expression levels of matrix metalloproteinase 9 and tissue inhibitor of metalloproteinase 3 via sirtuin 1 signaling in hydrogen peroxide-induced vascular smooth muscle cells
}

\author{
PENG LIU ${ }^{1}$, JIANFANG SU ${ }^{2}$, XIXI SONG ${ }^{3}$ and SHIXIAO WANG ${ }^{4}$ \\ ${ }^{1}$ Department of Cardiology, Shanghai East Hospital, Tongji University, Shanghai 200120; ${ }^{2}$ College of \\ Clinical Medicine, Shanxi Medical University, Taiyuan, Shanxi 030001; ${ }^{3}$ Department of Orthopaedics, \\ Shaanxi Provincial People's Hospital, Xi'an Jiaotong University, Xi'an, Shaanxi 710068; ${ }^{4}$ Department of \\ Internal Medicine, Shanghai Dermatology Hospital, Tongji University, Shanghai 200443, P.R. China
}

Received August 30, 2016; Accepted June 12, 2017

DOI: $10.3892 / \mathrm{mmr} .2017 .7937$

\begin{abstract}
Vascular smooth muscle cells (VSMCs) exhibit a notably increased rate of migration, which is one of the most common pathological changes in atherosclerosis. Investigations into the role of micro (mi)RNAs in the regulation of VSMC migration are beginning to emerge and additional miRNAs involved in VSMC migration modulation require identification. In the current study, VSMCs were primarily cultured from rat thoracic aortas, transfected with miR-92a mimics and induced by hydrogen peroxide $\left(\mathrm{H}_{2} \mathrm{O}_{2}\right)$ for $24 \mathrm{~h}$. Total mRNA and protein were collected for quantitative polymerase chain reaction and western blot analysis. In addition, the sirtuin 1 (SIRT1) gene was detected by luciferase reporter assay and VSMC migration was detected by Transwell migration assay. The current results demonstrated that reduced expression of miR-92a and overexpression of SIRT1 at the mRNA level were observed in $\mathrm{H}_{2} \mathrm{O}_{2}$-induced VSMCs. Furthermore, luciferase reporter assay demonstrated that the activity of the SIRT1 3'-untranslated region was reduced by miR-92a mimics. The upregulation of MMP9 and the downregulation of TIMP3 in $\mathrm{H}_{2} \mathrm{O}_{2}$-induced VSMCs were observed to be reversed by miR-92a mimics in addition to SIRT1 siRNA. Finally, Transwell migration assay revealed that miR-92a overexpression and silencing SIRT1 mitigated VSMC migration following $\mathrm{H}_{2} \mathrm{O}_{2}$ treatment. The present study indicated that $\mathrm{miR}-92 \mathrm{a}$ prevented the migration of $\mathrm{H}_{2} \mathrm{O}_{2}$-induced VSMCs by repressing the expression of
\end{abstract}

Correspondence to: Dr Shixiao Wang, Department of Internal Medicine, Shanghai Dermatology Hospital, Tongji University, 1278 Baode Road, Shanghai 200443, P.R. China

E-mail: wangshixiaosh@sina.com

Key words: microRNA-92a, matrix metalloproteinase 9, tissue inhibitor of metalloproteinase 3, sirtuin 1, vascular smooth muscle cells, hydrogen peroxide, migration
SIRT1, and also provided a novel therapy to protect against the phenotypic change of VSMCs in atherosclerosis.

\section{Introduction}

Atherosclerosis has been one of the most threatening types of diseases in the world, causing severe damage to health $(1,2)$. The migration of vascular smooth muscle cells (VSMCs), the most common pathological change in atherosclerosis, occurs in response to arterial injury and exerts a vital role in the development of atherosclerotic plaques $(3,4)$. Directional migration of VSMCs from the media to the intima through the basement membrane and collagenous matrix, and their subsequent proliferation are the key processes for self-remodeling of vessels (5). However, the molecular mechanism of abnormal VSMC phenotypic modulation in atherosclerosis is not fully understood.

Micro (mi)RNAs are a class of highly conserved small noncoding RNAs that regulate target genes by binding to the 3'-untranslated region (3'-UTR) of messenger RNA transcripts to repress their translation or mediate their degradation $(6,7)$. Certain critical roles of miRNAs in VSMC phenotypic change have previously been reported (8-11). Among the various miRNAs, miR-92a is a component of the miR-17-92 cluster, which is highly expressed in young endothelial cells in comparison with senescent endothelial cells, which exhibit increased oxidative stress and apoptosis (12). However, whether a miRNA-dependent mechanism contributes to hydrogen peroxide $\left(\mathrm{H}_{2} \mathrm{O}_{2}\right)$-induced changes in VSMCs remains unknown.

As reported previously, matrix metalloproteinases (MMPs) are major mediators of the vulnerability of atherosclerotic plaques. The balance between MMPs and tissue inhibitor of metalloproteinases (TIMPs) is important for the regulation of VSMC migration (13-16). It is well established that MMP9, inhibited by TIMP3, is a major molecular mediator that degrades the extracellular matrix (ECM), and detection of MMP9 supports the role of MMPs in vulnerable 
atherosclerotic plaques (17). High expression levels of MMP9 in atherosclerotic plaques contribute to plaque hemorrhage and rupture in specific MMP knockout mice and patients with acute coronary syndrome (18). However, the molecular mechanisms for MMP9/TIMP3 regulation in VSMCs remain elusive.

In order to investigate the possible effect of miR-92a and its underlying mechanisms on the migration of VSMCs in atherosclerosis, the present study utilized a cell model of $\mathrm{H}_{2} \mathrm{O}_{2}$-induced VSMCs obtained from rat thoracic aortas for experimental purposes.

\section{Materials and methods}

Experimental animals. A total of 20 male Sprague-Dawley (SD) rats (Specific-pathogen-free degree, aged 4-5 weeks, weight $100-150 \mathrm{~g}$ ) were purchased from Tongji University Laboratory Animal Center (Shanghai, China), and housed in a temperature-controlled feeding facility $\left(22^{\circ} \mathrm{C}\right)$ with a 12 -h light/dark cycle and free access to water and rodent diet. This study conformed to the Guide for Care and Use of Laboratory Animals and procedures were approved by the Animal Care and Use Committee of Tongji University School of Medicine (Shanghai, China).

Primary culture, identification and transfection of VSMCs. Primary VSMCs were isolated from SD rat thoracic aortas, as described previously (19). In brief, animals were sacrificed by intraperitoneal injection of $2 \%$ pentobarbital sodium (50 mg/kg; Sigma-Aldrich; Merck KGaA,Darmstadt, Germany) and perfused with saline for $5 \mathrm{~min}$. The thoracic aortas were aseptically harvested with removal of the intima, longitudinally cut into $\sim 1 \mathrm{~mm}^{2}$ fragments, explanted with the lumen side down on cell culture dishes (Corning Life Sciences, Beijing, China) containing Dulbecco's modified Eagle's medium (DMEM; Gibco; Thermo Fisher Scientific, Inc., Waltham, MA, USA) with $10 \%$ fetal bovine serum (FBS; Gibco; Thermo Fisher Scientific, Inc.) and maintained in a $37^{\circ} \mathrm{C}$ and humidified $5 \%$ $\mathrm{CO}_{2}$ incubator for 7 days, followed by discarding the tissue fragments and further collection of sprouted VSMCs. The following experiments were performed on the VSMCs between passages 4 and 7 in DMEM with 2.5\% FBS.

VSMCs were identified by their morphology and immunofluorescence detection of marker proteins, such as alpha-smooth muscle actin ( $\alpha$-SMA) using rabbit anti- $\alpha$-SMA polyclonal antibody (ab5694; 1:100; Abcam, Cambridge, UK), and their purity was ensured by multiple fluorescent stainings with $\alpha$-SMA antibody and nucleic acid dye, DAPI $(2 \mathrm{mg} / \mathrm{ml}$; Beyotime Institute of Biotechnology, Haimen, China) using a laser scanning confocal microscope (Leica Microsystems $\mathrm{GmbH}$, Wetzlar, Germany). To determine the effect of $\mathrm{H}_{2} \mathrm{O}_{2}$ on miR-92a expression in VSMCs, VSMCs were treated with $100 \mu \mathrm{M} \mathrm{H}_{2} \mathrm{O}_{2}$ (Sangon Biotech Co., Ltd., Shanghai, China) at $37^{\circ} \mathrm{C}$ for $24 \mathrm{~h}$.

For the transfection experiments, double-stranded miR-92a mimics or miRNA negative control (miR-NC; 100 nM; Shanghai GenePharma Co., Ltd., Shanghai, China), and sirtuin 1 (SIRT1) siRNA or NC siRNA (100 nM; Shanghai GenePharma Co., Ltd.) were respectively transfected into VSMCs using Lipofectamine ${ }^{\circledR} 2000$ reagent (Invitrogen; Thermo Fisher Scientific, Inc.) according to the manufacturer's protocols. Afterwards, VSMCs were cultured for $48 \mathrm{~h}$ and then used for subsequent assays.

Transwell migration assay. Cell migration assays were detected using a Transwell chamber with $8-\mu \mathrm{m}$ filter inserts (Corning Life Sciences, Beijing, China) without Matrigel. Following transfection and $\mathrm{H}_{2} \mathrm{O}_{2}$ treatment, $\sim 2 \times 10^{4}$ VSMCs in $100 \mu 1$ DMEM medium with $1 \%$ bovine serum albumin (BSA; Sangon Biotech Co., Ltd., Shanghai, China) were seeded into the upper chamber. The lower chamber was filled with $600 \mu \mathrm{l}$ DMEM medium containing 10\% FBS. After $24 \mathrm{~h}$, VSMCs on the upper chamber were gently scraped away, and the cells that had migrated through the filter into the lower chamber were fixed with 4\% paraformaldehyde (Sangon Biotech Co., Ltd.) for $15 \mathrm{~min}$ at room temperature and stained with DAPI $(2 \mathrm{mg} / \mathrm{ml})$. Then, the cells from 9 independent, randomly selected visual fields were counted under a laser scanning confocal microscope (magnification, x200; Leica Microsystems $\mathrm{GmbH}$ ) for quantitative analysis. Experiments were performed in triplicate.

Protein extraction and western blot analysis. Cellular protein was extracted with ice-cold radioimmunoprecipitation assay lysis buffer containing a mixture of proteasome inhibitors (Beyotime Institute of Biotechnology) for western blot analysis. The BCA method (BCA Protein Assay kit; Beyotime Institute of Biotechnology) was used to measure the protein concentrations of the supernatant by a microplate reader (BioTek Instruments, Inc., Winooski, VT, USA) according to the manufacturer's protocols. Equal amounts $(30 \mu \mathrm{g})$ of protein were then separated by $10 \%$ sodium dodecyl sulfate-polyacrylamide gel electrophoresis (SDS-PAGE) at $80 \mathrm{~V}$ for $0.5 \mathrm{~h}$ and subsequently $120 \mathrm{~V}$ for $1.5 \mathrm{~h}$ at room temperature, and transferred onto polyvinylidene fluoride membranes (EMD Millipore, Billerica, MA, USA) at $100 \mathrm{~V}$ for $1.5 \mathrm{~h}$ at $4^{\circ} \mathrm{C}$. The membranes were then blocked with 3\% BSA in phosphate-buffered saline (PBS; Sangon Biotech Co., Ltd.) for $30 \mathrm{~min}$ at room temperature and incubated with specific primary antibodies, including rabbit anti-MMP9 polyclonal antibody (ab38898; 1:1,000; Abcam), rabbit anti-TIMP3 monoclonal antibody (5673; 1:1,000; Cell Signaling Technology, Inc., Danvers, MA, USA), and mouse anti-GAPDH monoclonal antibody (97166; 1:1,000; Cell Signaling Technology, Inc.), overnight at $4^{\circ} \mathrm{C}$. Tris-buffered saline and Tween 20 (Sangon Biotech Co., Ltd.) was used to wash the membranes (3 times for $10 \mathrm{~min}$ ) and the membranes were incubated with secondary antibodies, including goat anti-rabbit immunoglobulin $\mathrm{G}(\mathrm{IgG})$-horseradish peroxidase (HRP) (L3012-2; 1:10,000; Signalway Antibody LLC, College Park, MD, USA), or goat anti-mouse IgG-HRP (L3032-2; 1:10,000; Signalway Antibody LLC), for $1 \mathrm{~h}$ at room temperature. The standard chemical luminescence method (BeyoECL Plus kit; Beyotime Institute of Biotechnology) was used to visualize the antigen by exposing the membranes to Kodak X-Omat AR film (Seebio Biotech, Inc., Shanghai, China) in accordance with the manufacturer's protocols. The resultant films were scanned on a gel imaging and analysis system and analyzed by Quantity One software version 4.4 (Bio-Rad Laboratories, Inc., Hercules, CA, USA). Relative protein expression of MMP9 and TIMP3 was normalized by the internal control, GAPDH, in the same sample. 
MTT assay. MTT assay was used to evaluate the effect of miR-92a mimics on VSMC viability. Briefly, following transfection and $\mathrm{H}_{2} \mathrm{O}_{2}$ treatment, VSMCs were cultured in DMEM medium in 96 -well plates at a density of 6,000 per well at $37^{\circ} \mathrm{C}$ under $5 \% \mathrm{CO}_{2}$. After $24 \mathrm{~h}$ of maintenance, $20 \mu \mathrm{lMTT}(5 \mathrm{mg} / \mathrm{ml}$ in PBS; Beyotime Institute of Biotechnology) was added to each plate and incubated for $4 \mathrm{~h}$ at $37^{\circ} \mathrm{C}$. Subsequently, the supernatant was removed and $150 \mu 1$ DMSO (Sangon Biotech Co., Ltd.) was added to each well. Following $10 \mathrm{~min}$ of incubation at room temperature, absorbance of formazan dissolved in DMSO was immediately assessed at a wavelength of $490 \mathrm{~nm}$ using a microplate reader (BioTek Instruments, Inc.). Experiments were performed in quintuplicate.

RNA extraction and quantitative polymerase chain reaction $(q P C R)$. Total RNA was isolated from VSMCs using TRIzol Reagent (Invitrogen; Thermo Fisher Scientific, Inc.) according to the manufacturer's protocols and its concentration was determined with NanoDrop Spectrophotometer (Thermo Fisher Scientific, Inc.). Reverse transcription of miRNA or ordinary RNA was performed using miRcute miRNA First-Strand cDNA Synthesis kit (Tiangen Biotech Co., Ltd., Beijing, China) or PrimeScript RT Reagent kit with gDNA Eraser (Perfect Real Time; Takara Biotechnology Co., Ltd., Dalian, China) according to the suggested protocol, respectively. Then, miR-92a expression level was measured with miRcute miRNA qPCR Detection kit (Tiangen Biotech Co., Ltd.) under the conditions: $94^{\circ} \mathrm{C}$ for $2 \mathrm{~min}$; 40 cycles $\left(94^{\circ} \mathrm{C}\right.$ for $20 \mathrm{sec}$ and $60^{\circ} \mathrm{C}$ for $34 \mathrm{sec}$ ), while the expression level of SIRT1 mRNA was evaluated with SYBR Green PCR Master Mix Kit (Takara Biotechnology Co., Ltd.) under the conditions: $95^{\circ} \mathrm{C}$ for $6 \mathrm{~min} ; 40$ cycles $\left(95^{\circ} \mathrm{C}\right.$ for $15 \mathrm{sec}$ and $60^{\circ} \mathrm{C}$ for $34 \mathrm{sec}$ ), using 7900 HT Real-Time PCR System (Applied Biosystems; Thermo Fisher Scientific, Inc.). U6 snRNA and GAPDH mRNA served as endogenous controls. The relative expression level was computed according to the $2^{-\Delta \Delta \mathrm{Cq}}$ analysis method (20). Experiments were performed in triplicate and the following primers were used: Forward, 5'-ACAGGCCGGGAC AAGTGCAATA-3' and reverse, 5'-GCTGTCAACGATACG CTACGTAACG-3' for miR-92a; forward, 5'-CTCGCTTCG GCAGCACA-3' and reverse, 5'-AACGCTTCACGAATTTGC GT-3' for U6; forward, 5'-CCAGATCCTCAAGCCATGT-3' and reverse, 5'-TTGGATTCCTGCAACCTG-3' for SIRT1; and forward, 5'-TGGGCTACACTGAGCACCAG-3' and reverse, 5'-AAGTGGTCGTTGAGGGCAAT-3' for GAPDH.

Luciferase reporter assay. Verification of miR-92a targeting SIRT1 was conducted by luciferase reporter assay in HEK-293T cells, as described previously (21). Briefly, wild-type 3'-UTR sequence of SIRT1 (WT-UTR) predicted to interact with miR-92a, was synthesized by GenePharma Co., Ltd and inserted into the dual-luciferase reporter vector (Promega Corporation, Madison, WI, USA), yielding the WT-UTR plasmid. Mutations (Mut-UTR) of potential miR-92a binding sites in WT-UTR were subjected to the Site-Directed Mutagenesis kit (Stratagene; Agilent Technologies, Inc., Santa Clara, CA, USA), generating the Mut-UTR plasmid. All plasmids were confirmed with DNA sequencing. HEK-293T cells were cultured in 12-well plates at $37^{\circ} \mathrm{C}$ under $5 \% \mathrm{CO}_{2}$ and cotransfected with the plasmids carrying $3^{\prime}$-UTR variants, and miR-92a mimics or miR-NC for
48 h, using Lipofectamine ${ }^{\circledR} 2000$ reagent (Invitrogen; Thermo Fisher Scientific, Inc.). Then, luciferase values of cell lysates were determined with a Dual-Luciferase Reporter Assay System (Promega Corporation) according to the protocol. All data were normalized by Renilla luciferase activity. Experiments were performed in triplicate.

Statistical analysis. All experiments were repeated 3 times. All statistical analyses were performed using SPSS version 20.0 (IBM Corp., Armonk, NY, USA). Data were presented as means \pm standard deviation. Student's t-test was used for comparisons between two different groups. One-way analysis of variance, followed by Tukey's post hoc test was performed to compare between multiple experimental groups. $\mathrm{P}<0.05$ was considered to indicate a statistically significant difference.

\section{Results}

Regulation of miR-92a expression level in VSMCs. In the present study, the expression level of miR-92a was decreased in VSMCs with $\mathrm{H}_{2} \mathrm{O}_{2}$ treatment for $24 \mathrm{~h}$ (Fig. 1A). This result intimated that miR-92a exerted a potential protective effect in VSMCs. Following transfection and $\mathrm{H}_{2} \mathrm{O}_{2}$ treatment, the expression of miR-92a in VSMCs was detected by qPCR (Fig. 1B). The outcomes revealed that the miR-92a expression level in the miR-92a mimic group was significantly higher than that in the miRNA negative control (miR-NC) group.

miR-92a overexpression inhibits the migration of VSMCs induced by $\mathrm{H}_{2} \mathrm{O}_{2}$ treatment. To investigate the potential effect of miR-92a on VSMC viability, cell viability was detected via MTT assay. It was found that $\mathrm{H}_{2} \mathrm{O}_{2}$ remarkably attenuated the viability of normal VSMCs. In contrast, cell viability in miR-92a mimic-transfected VSMCs was identified to be improved compared with miR-NC-transfected VSMCs following $\mathrm{H}_{2} \mathrm{O}_{2}$ treatment for $24 \mathrm{~h}$ (Fig. 1C).

In addition, cell migration assays were performed to evaluate whether miR-92a overexpression inhibited $\mathrm{H}_{2} \mathrm{O}_{2}$-induced VSMC migration. The Transwell migration assay demonstrated that the number of VSMCs in the $\mathrm{H}_{2} \mathrm{O}_{2}$ group that migrated through the membrane was greater compared with the control group. Notably, the miR-92a mimic group markedly decreased the number of VSMCs that migrated through the membrane when compared with the $\mathrm{H}_{2} \mathrm{O}_{2}$ group or the miR-NC group (Fig. 2A-E). These results illustrate that upregulated miR-92a expression by mimics suppresses $\mathrm{H}_{2} \mathrm{O}_{2}$-induced VSMC migration in the absence of adverse effects on cell viability.

miR-92a overexpression regulates the expression levels of MMP9 and TIMP3.miR-92a may target MMP9 and TIMP3, and influence their expression in certain types of cancer cells (22). However, the mechanism between miR-92 $\mathrm{a}$ and MMP9/TIMP3 in $\mathrm{H}_{2} \mathrm{O}_{2}$-induced VSMCs remains unclear. MMP9, inhibited by TIMP3, is involved in the progression of ECM degradation and VSMC migration. In the current study, the expression levels of MMP9 and TIMP3 in VSMCs following the above-mentioned transfection and $\mathrm{H}_{2} \mathrm{O}_{2}$ treatment were evaluated by western blot assay. The results demonstrated that $\mathrm{H}_{2} \mathrm{O}_{2}$ clearly caused the upregulation of MMP9 and the downregulation of TIMP3 in VSMCs. Nevertheless, transfection of miR-92a mimics 

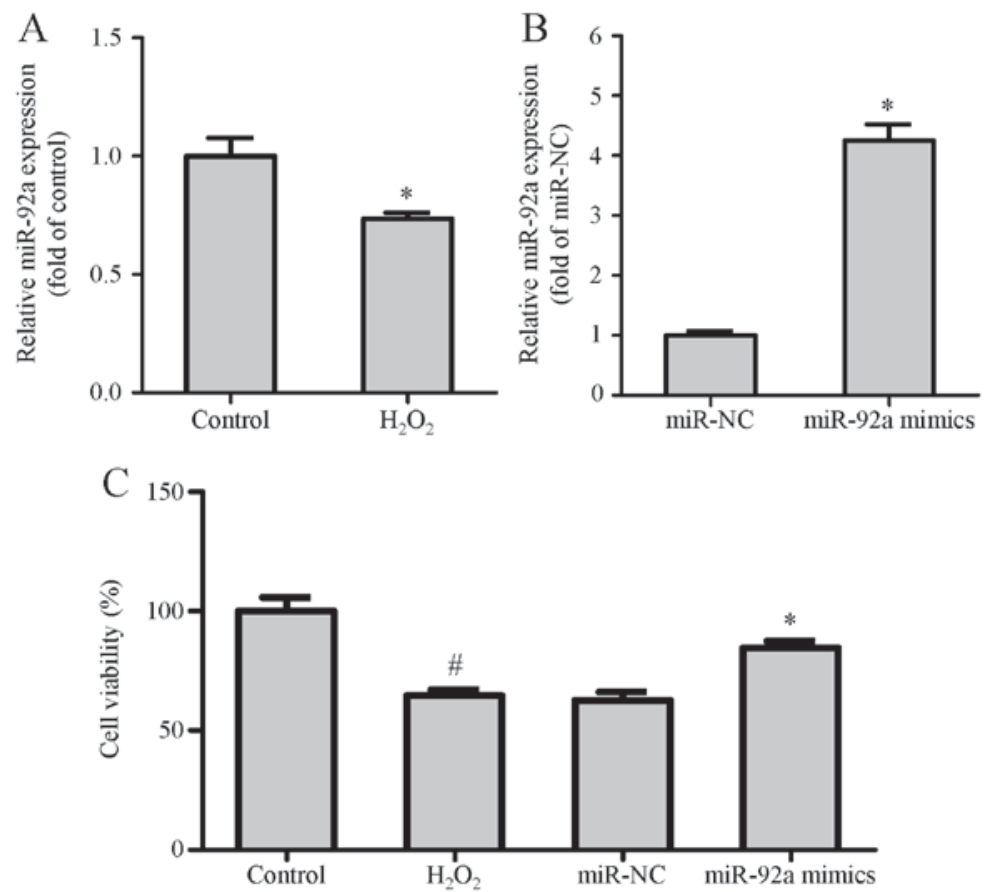

Figure 1. Regulation of miR-92a expression level in VSMCs. (A) Expression level of miR-92a in VSMCs was detected by qPCR, after $24 \mathrm{~h}$ of $\mathrm{H}_{2} \mathrm{O}_{2}$ treatment. Values are presented as means $\pm \mathrm{SD} ; \mathrm{n}=3$; ${ }^{*} \mathrm{P}<0.05$ vs. control group. (B) Transfection efficiency of miR-92a mimics in VSMCs was evaluated by qPCR, following a 24-h exposure to $\mathrm{H}_{2} \mathrm{O}_{2}$. Values are presented as means $\pm \mathrm{SD} ; \mathrm{n}=3 ;{ }^{*} \mathrm{P}<0.05$ vs. miR-NC group. (C) Viability of VSMCs was measured by MTT assay. Values are presented as means $\pm \mathrm{SD} ; \mathrm{n}=3 ;{ }^{*} \mathrm{P}<0.05$ vs. miR-NC group and ${ }^{\sharp} \mathrm{P}<0.05$ vs. control group. miR, microRNA; $\mathrm{H}_{2} \mathrm{O}_{2}$, hydrogen peroxide; $\mathrm{NC}$, negative control; VSMC, vascular smooth muscle cell; qPCR, quantitative polymerase chain reaction; SD, standard deviation.
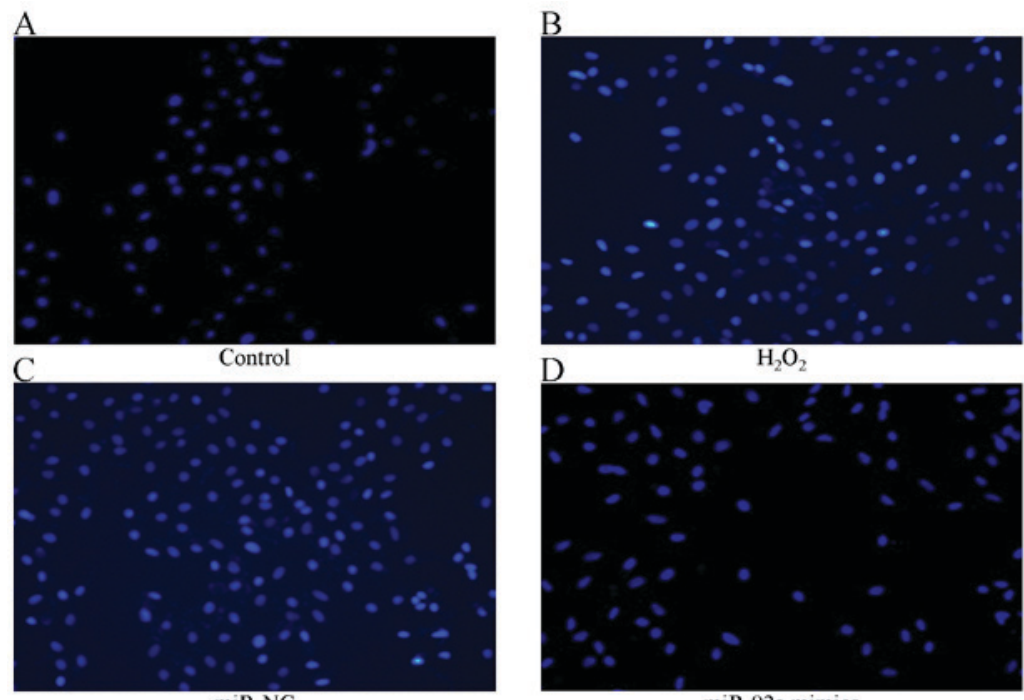

miR-NC

miR-92a mimics

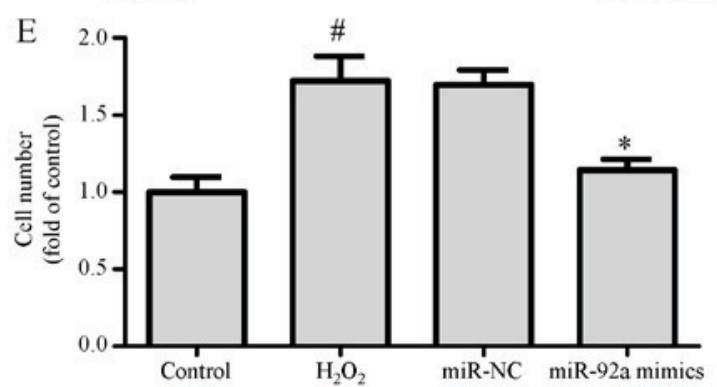

Figure 2. miR-92a overexpression inhibits $\mathrm{H}_{2} \mathrm{O}_{2}$-induced VSMC migration in vitro. Following transfection and $\mathrm{H}_{2} \mathrm{O}_{2}$ treatment, VSMCs were divided into four groups as follows: (A) Control, (B) $\mathrm{H}_{2} \mathrm{O}_{2}$, (C) miR-NC, (D) miR-92a mimics for Transwell migration assay, and (E) quantitative analysis of VSMCs vertically migrating into the lower chamber, based on DAPI staining and counting under confocal microscopy (magnification, x200). Values are presented as means $\pm \mathrm{SD} ; \mathrm{n}=3$; ${ }^{*} \mathrm{P}<0.05$ vs. miR-NC group and ${ }^{\#} \mathrm{P}<0.05$ vs. control group. miR, microRNA; $\mathrm{H}_{2} \mathrm{O}_{2}$, hydrogen peroxide; $\mathrm{NC}$, negative control; VSMC, vascular smooth muscle cell; SD, standard deviation. 


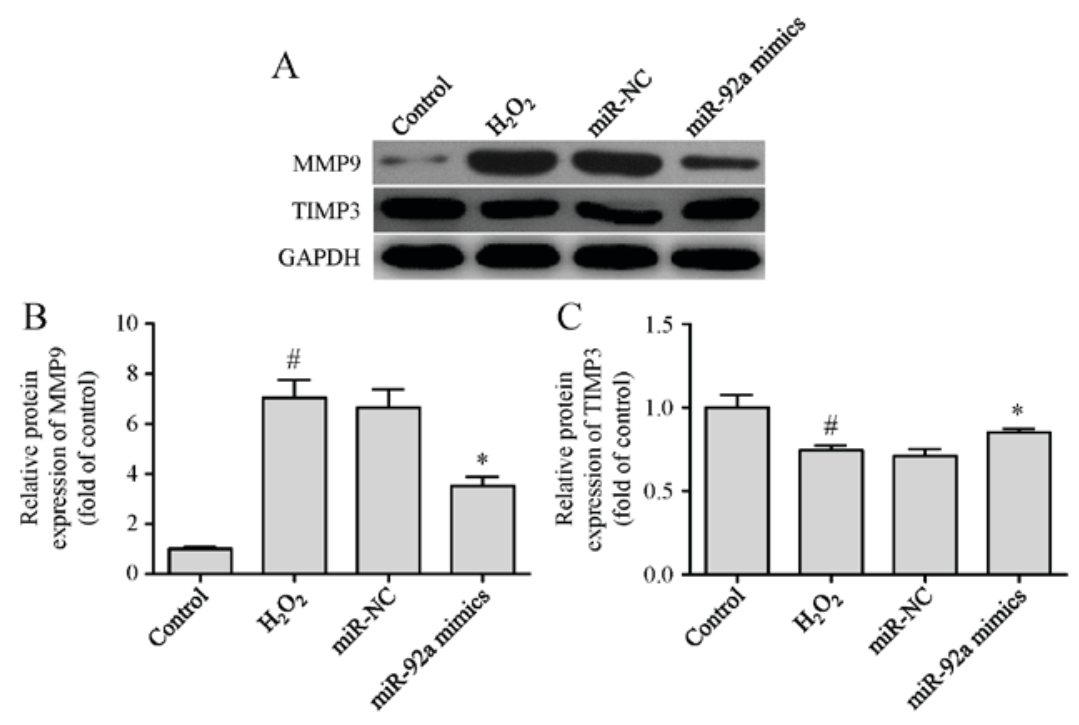

Figure 3. miR-92a overexpression regulates the expression levels of MMP9 and TIMP3. VSMCs were transfected with miR-92a mimics or miR-NC, induced by $\mathrm{H}_{2} \mathrm{O}_{2}$, and further processed for (A) western blot analysis of MMP9 and TIMP3 protein expression levels, and densitometric analysis of (B) MMP9 and (C) TIMP3 protein expression levels normalized to GAPDH, in each group. Values are presented as means $\pm \mathrm{SD}$; $\mathrm{n}=3$; " $\mathrm{P}<0.05$ vs. miR-NC group and ${ }^{\text {"}} \mathrm{P}<0.05$ vs. control group. miR, microRNA; $\mathrm{H}_{2} \mathrm{O}_{2}$, hydrogen peroxide; $\mathrm{NC}$, negative control; MMP9, matrix metalloproteinase 9; TIMP3, tissue inhibitor of metalloproteinase 3; VSMC, vascular smooth muscle cell; SD, standard deviation.

markedly decreased the expression level of MMP9, but clearly increased the expression level of TIMP3 in comparison with transfection of miR-NC in $\mathrm{H}_{2} \mathrm{O}_{2}$-treated VSMCs (Fig. 3A-C). These data suggest that the expression levels of MMP9 and TIMP3 are modulated by miR-92a in VSMCs.

SIRT1 is a direct target of miR-92a. To verify whether miR-92a directly targets the SIRT1 gene, dual-luciferase reporter vectors harboring a wild-type or mutant 3'-UTR region of SIRT1 were constructed and transfected into HEK-293T cells. Dual-luciferase reporter assay demonstrated that miR-92a mimics significantly weakened the luciferase activity of the reporter vector containing a wild-type SIRT1 3'-UTR sequence (WT-UTR) compared with miR-NC. However, this inhibitive effect of miR-92a mimics was eliminated when the reporter vector contained a mutant SIRT1 3'-UTR sequence (Mut-UTR), which was unable to interact with miR-92a (Fig. 4A and B). These results indicate that miR-92a directly binds to SIRT1.

Silencing SIRT1 regulates the expression levels of MMP9 and TIMP3 and inhibits the migration of VSMCs induced by $\mathrm{H}_{2} \mathrm{O}_{2}$ treatment. The above-mentioned findings suggested that miR-92a may regulate the expression levels of MMP9 and TIMP3 to inhibit VSMC migration by targeting SIRT1. To ascertain whether this influence can be indicated by silencing SIRT1, SIRT1 siRNA was transfected into VSMCs before $\mathrm{H}_{2} \mathrm{O}_{2}$ treatment. The transfection efficiency of SIRT1 siRNA and its influence on the expression levels of MMP9 and TIMP 3 were demonstrated by qPCR and western blot analysis, respectively. The data revealed that the overexpression of SIRT1 mRNA resulting from $\mathrm{H}_{2} \mathrm{O}_{2}$ stimulation was markedly reduced in the SIRT1-siRNA group compared with the NC-siRNA group (Fig. 5A). As presented in Fig. 5B-D, SIRT1 siRNA decreased the expression level of MMP9, and increased the expression level of TIMP3 in $\mathrm{H}_{2} \mathrm{O}_{2}$-treated

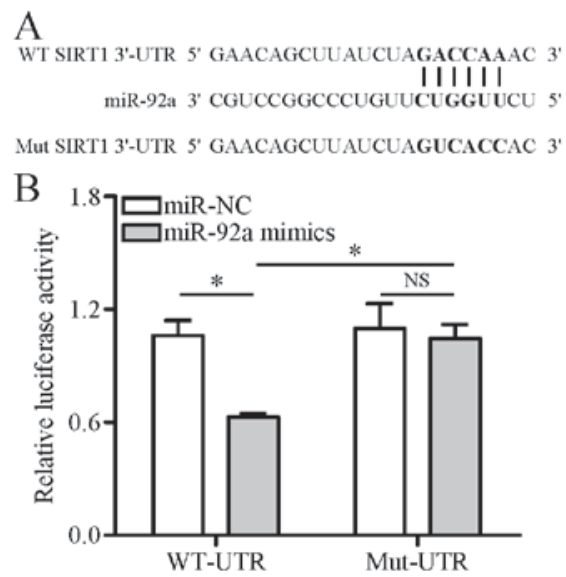

Figure 4. SIRT1 is directly targeted by miR-92a. (A) Sequence alignments of WT and Mut predicted miR-92a binding sites in the 3'-UTR of SIRT1 mRNA. (B) Direct combination of miR-92a and SIRT1 was examined by dual-luciferase reporter assay in HEK-293T cells. Data shown that miR-92a repressed the luciferase activity of WT SIRT1 3'-UTR (WT-UTR), while exhibited no effect on that of Mut SIRT1 3'-UTR (Mut-UTR). Values are presented as means $\pm \mathrm{SD} ; \mathrm{n}=3 ;{ }^{*} \mathrm{P}<0.05$. SIRT1, sirtuin $1 ; 3$ '-UTR, 3'-untranslated region; WT, wild-type; Mut, mutant; miR, microRNA; NC, negative control; NS, no significance; SD, standard deviation.

VSMCs. Notably, the Transwell migration assay demonstrated that the quantity of VSMCs in the SIRT1-siRNA group that migrated via the membrane was lower than that in the $\mathrm{H}_{2} \mathrm{O}_{2}$ group or the NC-siRNA group (Fig. 6A-E). Collectively, these outcomes demonstrated that miR-92a regulates the expression levels of MMP9 and TIMP3 via SIRT1 signaling, and further interferes with $\mathrm{H}_{2} \mathrm{O}_{2}$-induced VSMC migration.

\section{Discussion}

In the current study, miR-92a was demonstrated to be capable of suppressing migration in $\mathrm{H}_{2} \mathrm{O}_{2}$-treated VSMCs. To fully 

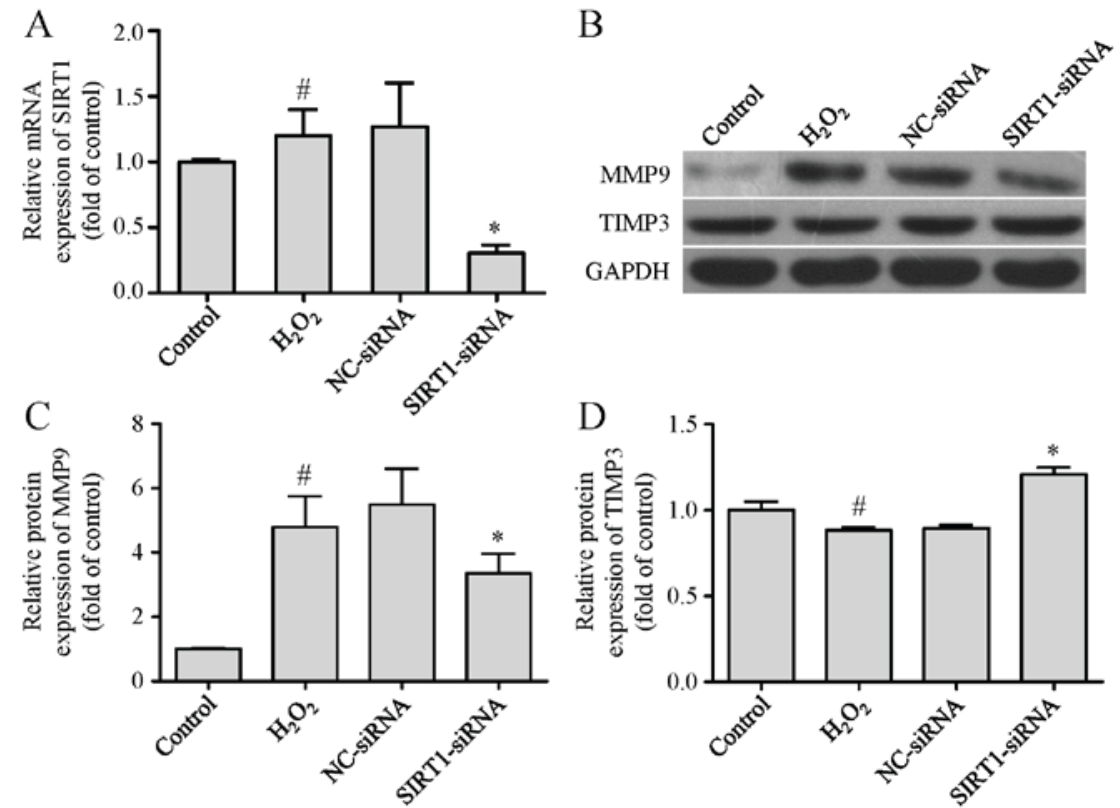

Figure 5. Silencing SIRT1 regulates the expression levels of MMP9 and TIMP3. VSMCs were transfected with SIRT1-siRNA or NC-siRNA, induced by $\mathrm{H}_{2} \mathrm{O}_{2}$, and further processed for (A) qPCR analysis of SIRT1 mRNA expression level normalized to GAPDH, (B) western blot analysis of MMP9 and TIMP3 protein expression levels, and densitometric analysis of (C) MMP9 and (D) TIMP3 protein expression levels normalized to GAPDH, in each group. Values are presented as means $\pm \mathrm{SD} ; \mathrm{n}=3 ;{ }^{*} \mathrm{P}<0.05$ vs. NC-siRNA group and ${ }^{*} \mathrm{P}<0.05$ vs. control group. SIRT1, sirtuin $1 ; \mathrm{H}_{2} \mathrm{O}_{2}$, hydrogen peroxide; $\mathrm{NC}$, negative control; MMP9, matrix metalloproteinase 9; TIMP3, tissue inhibitor of metalloproteinase 3; VSMC, vascular smooth muscle cell; qPCR, quantitative polymerase chain reaction; $\mathrm{SD}$, standard deviation.
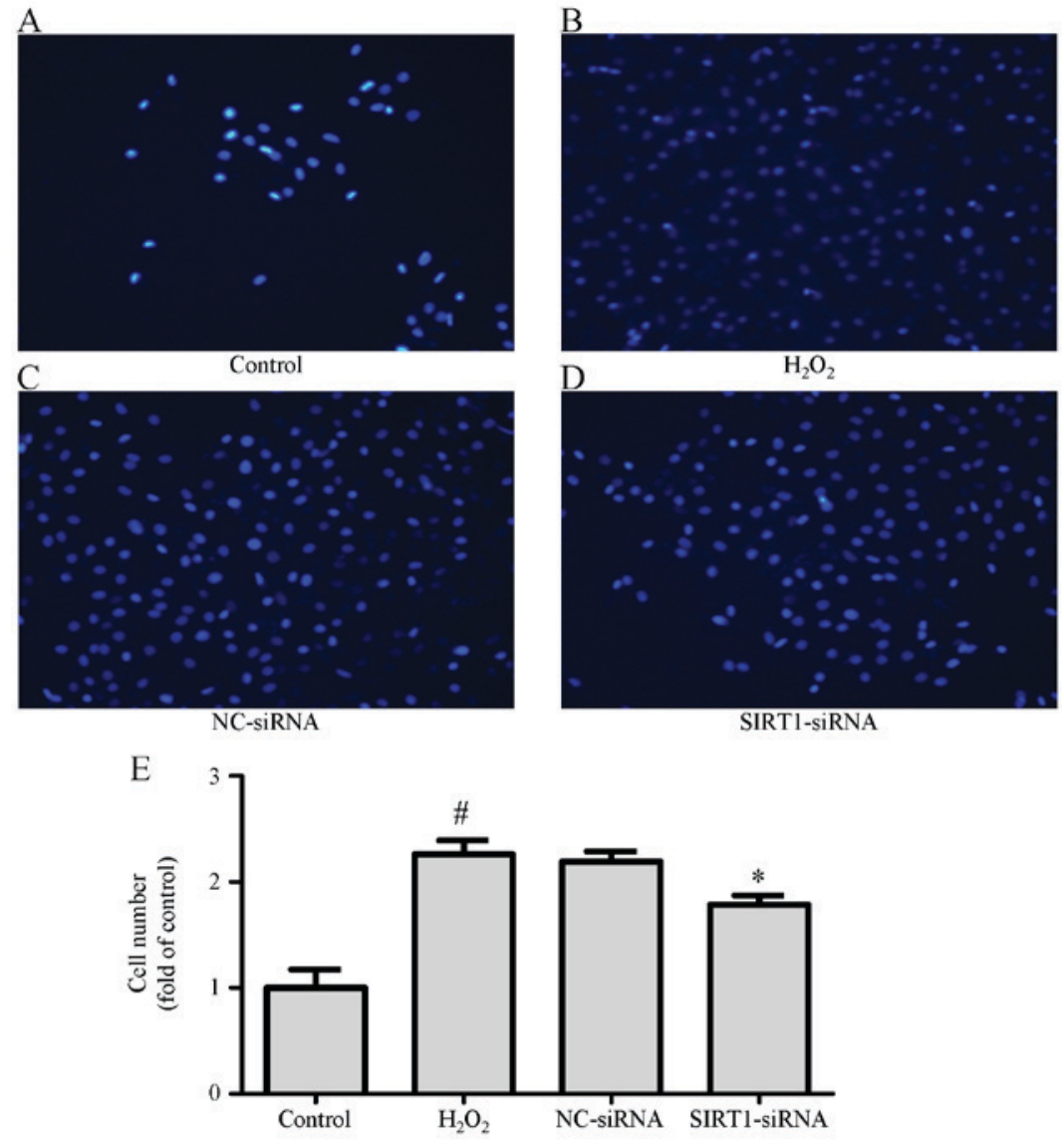

Figure 6. Silencing SIRT1 inhibits $\mathrm{H}_{2} \mathrm{O}_{2}$-induced VSMC migration in vitro. Following transfection and $\mathrm{H}_{2} \mathrm{O}_{2}$ treatment, VSMCs were divided into four groups as follows: (A) Control, (B) $\mathrm{H}_{2} \mathrm{O}_{2}$, (C) NC-siRNA, (D) SIRT1-siRNA for Transwell migration assay, and (E) quantitative analysis of VSMCs vertically migrating into the lower chamber, based on DAPI staining and counting under confocal microscopy (magnification, $\mathrm{x} 200$ ). Values are presented as means $\pm \mathrm{SD}$; $\mathrm{n}=3$; ${ }^{*} \mathrm{P}<0.05$ vs. NC-siRNA group and ${ }^{\#} \mathrm{P}<0.05$ vs. control group. SIRT1, sirtuin $1 ; \mathrm{H}_{2} \mathrm{O}_{2}$, hydrogen peroxide; $\mathrm{NC}$, negative control; VSMC, vascular smooth muscle cell; SD, standard deviation. 
investigate the role of miR-92a and the underlying mechanisms in injured VSMCs, a model of $\mathrm{H}_{2} \mathrm{O}_{2}$-treated VSMCs was established, and the downregulation of miR-92a was demonstrated by this model, which was consistent with the study by Zhang et al (10). The migration of VSMCs is known to be one of the essential prerequisites for atherogenesis following arterial injury (4). In the present study, the overexpression of miR-92a by mimics was confirmed to inhibit VSMC migration and regulate MMP9 and TIMP3 expression levels via SIRT1 signaling.

miRNAs are engaged in a variety of biological processes and multiple pathogenic events, including cell migration, proliferation, inflammation, oxidation, and apoptosis $(23,24)$. As reported previously, miR-92a overexpression inhibits $\mathrm{H}_{2} \mathrm{O}_{2}$-induced VSMC apoptosis by directly targeting the mitogen-activated protein kinase kinase 4-c-Jun N-terminal kinase 1 signaling pathway (10). In the current study, miR-92a overexpression suppressed cell migration by inhibiting the ratio of MMP9/TIMP3 in $\mathrm{H}_{2} \mathrm{O}_{2}$-induced VSMCs. Of note, VSMC migration is known to be an indispensable element in the incidence and progression of atherosclerotic lesions (25). Additionally, MMPs and TIMPs together maintain the balance of the ECM, and contribute to the regulation of VSMC migration $(14,26)$. A previous study identified that the significant decrease of TIMP3 expression levels in human carotid atherosclerotic plaques associated with type 2 diabetes mellitus, exhibit a high correlation with MMP9 overactivity in these subjects (27). Therefore, the present data suggested that miR-92a overexpression is considered to be an important potential target for therapeutic intervention of atherosclerosis.

To further explain the association by which miR-92a action affects VSMC migration, the expression level of SIRT1 mRNA, potentially interacting with miR-92a, was measured in $\mathrm{H}_{2} \mathrm{O}_{2}$-induced VSMCs. In the present study, $\mathrm{H}_{2} \mathrm{O}_{2}$ decreased mRNA expression levels of miR-92a but increased that of SIRT1 in VSMCs, suggesting that VSMC migration was related to the expression changes of SIRT1 targeted by miR-92a. SIRT1, a member of the sirtuin family (SIRT1-7) or silent information regulator 2 proteins, performs various biological functions in the development of cardiovascular diseases (28). Cardellini et al (27) indicated that SIRT1 signaling was involved in regulating MMP9/TIMP3 activity of diabetic atherosclerosis. Furthermore, it has been proposed that SIRT1 is a downstream target of miRNAs in vascular injury (29-31). In the current study, it was identified that SIRT1 siRNA played a similar role with miR-92a mimics in the regulation of MMP9 and TIMP3. Specifically, SIRT1 siRNA attenuated the expression level and secretion of MMP9, and promoted TIMP3 expression by knockdown of SIRT1 gene in $\mathrm{H}_{2} \mathrm{O}_{2}$-induced VSMCs. Moreover, SIRT1 was successfully demonstrated as a target gene of miR-92a in a dual-luciferase reporter assay and silencing SIRT1 did not result in a migration-promoting effect in vitro, which was also similar to miR-92a overexpression. Taken together, the results indicate that miR-92a decreases the expression level of MMP9 and increases the expression level of TIMP3 via SIRT1 signaling, which accounts for the inhibition of VSMC migration.

In conclusion, miR-92a overexpression modulates MMP9 and TIMP3 expression levels and prevents $\mathrm{H}_{2} \mathrm{O}_{2}$-induced VSMC migration by repressing SIRT1, implying that miR-92a may exert an atheroprotective role in atherogenesis. Therefore, upregulation of miR-92a expression may present a novel therapeutic strategy in protecting against the dysfunction of VSMCs in atherosclerosis.

\section{Acknowledgements}

The authors would like to thank Mr. Jun Tian (The Second Military Medical University, Shanghai, China) for data analysis in the current research, and Miss Zhen Guo (Pfeiffer University, Misenheimer, NC, USA) for language modification of the manuscript.

\section{References}

1. Libby P, Ridker PM and Hansson GK: Progress and challenges in translating the biology of atherosclerosis. Nature 473: 317-325, 2011.

2. Verweij SL, van der Valk FM and Stroes ES: Novel directions in inflammation as a therapeutic target in atherosclerosis. Curr Opin Lipidol 26: 580-585, 2015.

3. Stringa E, Knäuper V, Murphy G and Gavrilovic J: Collagen degradation and platelet-derived growth factor stimulate the migration of vascular smooth muscle cells. J Cell Sci 113 (Pt 11): 2055-2064, 2000.

4. Rudijanto A: The role of vascular smooth muscle cells on the pathogenesis of atherosclerosis. Acta Med Indones 39: 86-93, 2007.

5. Zhang J, Zou F, Tang J, Zhang Q, Gong Y, Wang Q, Shen Y, Xiong L, Breyer RM, Lazarus M, et al: Cyclooxygenase-2-derived prostaglandin $\mathrm{E}_{2}$ promotes injury-induced vascular neointimal hyperplasia through the E-prostanoid 3 receptor. Circ Res 113: 104-114, 2013.

6. Albinsson S and Sessa WC: Can microRNAs control vascular smooth muscle phenotypic modulation and the response to injury? Physiol Genomics 43: 529-533, 2011.

7. Robinson $\mathrm{HC}$ and Baker AH: How do microRNAs affect vascular smooth muscle cell biology? Curr Opin Lipidol 23: 405-411, 2012.

8. Ning B, Gao L, Liu RH, Liu Y, Zhang NS and Chen ZY: microRNAs in spinal cord injury: Potential roles and therapeutic implications. Int J Biol Sci 10: 997-1006, 2014.

9. Chio CC, Lin JW, Cheng HA, Chiu WT, Wang YH, Wang JJ, Hsing $\mathrm{CH}$ and Chen RM: MicroRNA-210 targets antiapoptotic $\mathrm{Bcl}-2$ expression and mediates hypoxia-induced apoptosis of neuroblastoma cells. Arch Toxicol 87: 459-468, 2013.

10. Zhang L, Zhou M, Wang Y, Huang W, Qin G, Weintraub NL and Tang Y: miR-92a inhibits vascular smooth muscle cell apoptosis: Role of the MKK4-JNK pathway. Apoptosis 19: 975-983, 2014.

11. Lv H, Zhang Z, Wang Y, Li C, Gong W and Wang X: MicroRNA-92a promotes colorectal cancer cell growth and migration by inhibiting KLF4. Oncol Res 23: 283-290, 2016.

12. Rippe C, Blimline M, Magerko KA, Lawson BR, LaRocca TJ, Donato AJ and Seals DR: MicroRNA changes in human arterial endothelial cells with senescence: Relation to apoptosis, eNOS and inflammation. Exp Gerontol 47: 45-51, 2012.

13. Lenglet S, Thomas A, Chaurand P, Galan K, Mach F and Montecucco F: Molecular imaging of matrix metalloproteinases in atherosclerotic plaques. Thromb Haemost 107: 409-416, 2012.

14. Zhao B, Luo X, Shi H and Ma D: Tissue factor pathway inhibitor-2 is downregulated by ox-LDL and inhibits ox-LDL induced vascular smooth muscle cells proliferation and migration. Thromb Res 128: 179-185, 2011.

15. Vigetti D, Moretto P, Viola M, Genasetti A, Rizzi M, Karousou E, Pallotti F, De Luca G and Passi A: Matrix metalloproteinase 2 and tissue inhibitors of metalloproteinases regulate human aortic smooth muscle cell migration during in vitro aging. FASEB J 20: 1118-1130, 2006.

16. Chen Q, Jin M, Yang F, Zhu J, Xiao Q and Zhang L: Matrix metalloproteinases: Inflammatory regulators of cell behaviors in vascular formation and remodeling. Mediators Inflamm 2013: 928315, 2013

17. Halade GV, Jin YF and Lindsey ML: Matrix metalloproteinase (MMP)-9: A proximal biomarker for cardiac remodeling and a distal biomarker for inflammation. Pharmacol Ther 139: 32-40, 2013. 
18. Hobeika MJ, Thompson RW, Muhs BE, Brooks PC and Gagne PJ: Matrix metalloproteinases in peripheral vascular disease. J Vasc Surg 45: 849-857, 2007.

19. Ha JM, Yun SJ, Jin SY, Lee HS, Kim SJ, Shin HK and Bae SS: Regulation of vascular smooth muscle phenotype by cross-regulation of krüppel-like factors. Korean J Physiol Pharmacol 21: 37-44, 2017.

20. Livak KJ and Schmittgen TD: Analysis of relative gene expression data using real-time quantitative PCR and the 2(-Delta Delta C(T)) method. Methods 25: 402-408, 2001.

21. Yu J, Wang L, Yang H, Ding D, Zhang L, Wang J, Chen Q, Zou Q, Jin Y and Liu X: Rab14 suppression mediated by MiR-320a Inhibits cell proliferation, migration and invasion in breast cancer. J Cancer 7: 2317-2326, 2016.

22. Jo DH, Kim JH, Cho CS, Cho YL, Jun HO, Yu YS, Min JK and Kim JH: STAT3 inhibition suppresses proliferation of retinoblastoma through down-regulation of positive feedback loop of STAT3/miR-17-92 clusters. Oncotarget 5: 11513-11525, 2014.

23. Hammond SM: An overview of microRNAs. Adv Drug Deliv Rev 87: 3-14, 2015.

24. McManus DD and Freedman JE: MicroRNAs in platelet function and cardiovascular disease. Nat Rev Cardiol 12: 711-717, 2015.

25. Zhou Y, Zhang MJ, Li BH, Chen L, Pi Y, Yin YW, Long CY, Wang X, Sun MJ, Chen X, et al: PPAR $\gamma$ inhibits VSMC proliferation and migration via attenuating oxidative stress through upregulating UCP2. PLoS One 11: e0154720, 2016.
26. Shi YF, Chi JF, Tang WL, Xu FK, Liu LB, Ji Z, Lv HT and Guo HY: Effects of rosuvastatin on the production and activation of matrix metalloproteinase-2 and migration of cultured rat vascular smooth muscle cells induced by homocysteine. J Zhejiang Univ Sci B 14: 696-704, 2013.

27. Cardellini M, Menghini R, Martelli E, Casagrande V, Marino A, Rizza S, Porzio O, Mauriello A, Solini A, Ippoliti A, et al: TIMP3 is reduced in atherosclerotic plaques from subjects with type 2 diabetes and increased by SirT1. Diabetes 58: 2396-2401, 2009.

28. Ma L and Li Y: SIRT1: Role in cardiovascular biology. Clin Chim Acta 440: 8-15, 2015.

29. Volkmann I, Kumarswamy R, Pfaff N, Fiedler J, Dangwal S, Holzmann A, Batkai S, Geffers R, Lother A, Hein L and Thum T: MicroRNA-mediated epigenetic silencing of sirtuin1 contributes to impaired angiogenic responses. Circ Res 113 997-1003, 2013.

30. Menghini R, Casagrande V, Cardellini M, Martelli E, Terrinoni A, Amati F, Vasa-Nicotera M, Ippoliti A, Novelli G, Melino G, et al: MicroRNA 217 modulates endothelial cell senescence via silent information regulator 1. Circulation 120: 1524-1532, 2009

31. Kumarswamy R, Volkmann I, Beermann J, Napp LC, Jabs O, Bhayadia R, Melk A, Ucar A, Chowdhury K, Lorenzen JM, et al: Vascular importance of the miR-212/132 cluster. Eur Heart J 35: 3224-3231, 2014 